\title{
Educación inclusiva. Sonrisas y lágrimas
}

\author{
Gerardo Echeita Sarrionandia
}

Departamento Interfacultativo de Psicología Evolutiva y de la Educación Universidad Autónoma de Madrid

\section{RESUMEN}

En este artículo se analiza el significado global que tiene el derecho a una educación inclusiva, que internacionalmente está formalmente establecido. Al hilo de algunas preguntas básicas sobre esta cuestión y de sus respectivas respuestas, se articula el análisis sobre el sentido, las dimensiones, y dilemas a los que hoy se enfrentan los agentes educativos encargados de implementarla, en particular en España, si bien muchos de ellos son similares en otras partes del mundo. La contradicción fragrante entre lo que se dice en las normas y lo que realmente ocurre en muchos centros educativos, "entre lo dicho y lo hecho", genera enormes tensiones y desgarros emocionales que afectan muy negativamente a muchos estudiantes vulnerables y a sus familias.

Palabras Clave: educación inclusiva, derecho, dilemas,contradicciones.

\section{Educación inclusiva. Sonrisas y lágrimas}

\section{ABSTRACT}

This paper discusses the overall meaning which has the right to inclusive education that internationally is formally established. The analysis of the dimensions regarding inclusive education, besides the dilemmas faced by educational stakeholders responsible for implementing, its organised trough some basic questions and their answer. This analysis is relevant for the Spanish context but also in other countries around de world. The big gap among theory and practices, create enormous tensions and emotional tears that affect very negatively to many vulnerable students and their families.

Keywords: inclusive education, rigths, dilemmas, contradictions.

\section{Introducción.}

Estamos de lleno en el proceso de analizar y debatir en el Congreso de los Diputados y fuera de él, una nueva ley de educación en nuestro país. En este texto presento lo fundamental de la comparecencia que he podido realizar ante la Subcomisión del parlamento que está organizando los trabajos preparatorios para dicho proyecto de ley.

Mi aportación a ese debate se ha centrado en la tarea de señalar la importancia de que la nueva ley de educación responda al compromiso inequívoca y al desafío de tratar de garantizar la equidad educativa, condición necesaria para conseguir, con y por ello, que la educación escolar sea más inclusiva.

Comparto con otros colegas, dentro y fuera de nuestro país (Ainscow, 2016; Echeita, Martín, Simón y Sandoval, 2016), que hablar de "educación inclusiva" no es sino una perspectiva desde la que analizar los desafíos de la equidad en la educación escolar $\mathrm{y}$, por lo tanto, una aspiración inserta en ese principio general. En todo caso, lo que está claro que esa aspiración de mayor equidad no se conseguirá sin que los países cumplan el mandato que han recibido de que sus sistemas educativos sean inclusivos.
Y hablo de "mandato" no en términos retóricos, sino porque ese es el compromiso adquirido y comprometido formalmente por España, ante varios organismos internacionales. El primero ante la UNESCO, a la cual debemos reconocer su rol de señalamiento del horizonte hacia el cual deben orientarse las políticas educativas de los "Estados parte". A este respecto señalaré dos referencias recientes ineludibles: lo acordado en la 48 Sesión de la Conferencia Internacional de Educación promovida por UNESCO/BIE (2008), con el elocuente título de: "La educación inclusiva: el camino hacia el futuro". La segunda (UNESCO et al, 2016), la llamada Declaración de Incheon y su Marco de Acción para la realización del Objetivo de Desarrollo Sostenible 4, en el año 2030, que de nuevo tiene un título relevante para lo que nos ocupa: "Garantizar una educación inclusiva y equitativa de calidad y promover oportunidades de aprendizaje permanente para todos".

Como trataré de explicar, la educación inclusiva no es una aspiración que se refiera exclusivamente a una determinada población escolar, en particular a la de los niños y niñas (también jóvenes y adultos) en situación de (dis)capacidad o con dificultades en su aprendizaje de distinta índole. Se trata de una meta que quiere ayudar a transformar los sistemas educativos para que 
TODO el alumnado, sin restricciones, limitaciones ni eufemismos respecto a ese TODOS, tenga oportunidades equiparables y de calidad para el desarrollo pleno de su personalidad, fin último de todos los sistemas educativos. Pero es evidente, al mismo tiempo, que el alumnado con necesidades de apoyo educativo especificas (siguiendo la categorización establecida en la LOMCE), está en mayor riesgo de segregación, marginación o fracaso escolar y por ello, es justo, prestar especial atención a que sus derechos no queden relegados.

En este marco, los "Estados parte" también han recibido el mandato del Comité encargado dentro del sistema de Naciones Unidas del seguimiento de la Convención de los Derechos de las Personas con Discapacidad (UN, 2006), ratificada por España en 2008, de velar por el cabal cumplimiento del "derecho a una educación inclusiva" (Art. 24 de la Convención). Para facilitar la tarea de compartir un marco de referencia común de lo que cabe entender como educación inclusiva, el Comité encargado del seguimiento de dicha Convención ha elaborado una Observación o Comentario General (la no 4) (UN, 2016), que explica y detalla el significado y alcance de este derecho para los países signatarios, con el valor añadido de ser una interpretación autorizada y legítima.

Este compromiso adquirido al firmar la Convención de los Derechos de las Personas con Discapacidad, es un hecho muy relevante, porque bien podría decirse que cambia el estatus de esta preocupación; de ser entendida como un principio bien intencionado y aplicable, digamos, "hasta donde fuera razonable", ahora es un derecho, con toda su fuerza jurídica y social (Campoy, 2007; Lema, 2009), y para el cual se puede y se debe pedir el amparo del sistema judicial, algo que, organizaciones como la Fundación Gerard $^{1}$ o Solcom ${ }^{2}$ ya realizan.

Esta perspectiva de los derechos, confiere a los análisis que nos ocupan un matiz muy relevante que no se debe desdeñar, pues si se llegaran a hacer propuestas en la ley contrarias al sentido de este derecho a una educación inclusiva, bien cabría considerarlas como actos, directos o indirectos, de discriminación (Lema, 2009).

Finalmente, y en el marco de los Objetivos para el Desarrollo Sostenible 2030, impulsados nuevamente por Naciones Unidas (2016) con la esperanza de "que los países y los ciudadanos del mundo emprendan un nuevo camino para mejorar las vidas de las personas en todas partes", hay que destacar que, como ya se ha señalado, el Objetivo 4 vuelve a resaltar la importancia estratégica de trabajar para; "Garantizar una educación inclusiva, equitativa y de calidad y promover oportunidades de aprendizaje durante toda la vida para todos".

A la vista de estos compromisos, libremente adoptados parece claro que es necesario interrogarnos sobre el sentido, el alcance y las implicaciones de este enfoque que debemos dar a la educación escolar para que sea "inclusiva", y que la nueva ley de educación que se está preparando no puede obviar.

En adelante, articularé este texto alrededor de algunas preguntas básicas, cuyas respuestas creo que pueden ayudar a clarificar por qué, y de quién hablamos cuando hablamos de educación inclusiva y a entender mejor los argumentos que nos llevan hacia ella, así como comprender su naturaleza y la de algunos de los enormes desafíos que su implementación nos plantea a todos. Ya anticipo que lo sustantivo y más importante de todas esas preguntas/respuestas alrededor de la educación inclusiva, es que lo que estamos tratando es un asunto de carácter político que versa sobre el proyecto de sociedad en el que queremos vivir (Echeita, 2013; Slee, 2012).
Y con relación a ese proyecto social una dimensión, sin duda alguna muy relevante, tiene que ver con la pregunta de Alain Touraine (2005) "¿Podremos vivir juntos?" y que debemos completar con otras igualmente incómodas; ¿Cómo queremos hacerlo?, ¿De forma que se garantice la equidad y el respeto a la diversidad humana en un marco de derechos y deberes compartido? o ¿Mirando indiferentes hacia otro lado ante las injustas desigualdades tantas veces asociadas a factores como el género, el origen social, la capacidad, la orientación afectivo sexual o el territorio donde uno vive? Según sea de clara y contundente (o ambigua) la respuesta que el cuerpo social dé a estas preguntas, así será de claro y contundente (o ambiguo) el mandato que recibirá "la escuela" (en una acepción amplia del término), y otros agentes educativos, para que su acción educativa sea coherente con el horizonte social señalado.

No creo que nadie se extrañará si digo que el mandato recibido por "la escuela" hasta hace muy poco tiempo no es el propio de quienes han querido una sociedad inclusiva, sino más bien, el de quienes han querido y se han beneficiado de una sociedad estratificada, segregada y desigual. Ahora bien, a la vista de algunos acontecimientos políticos recientes (elecciones en EE. UU, Holanda, Francia,...) todo apunta a que no hablamos solo "del pasado" sino también de un presente muy inquietante.

\section{2. ¿Por qué hablamos de educación inclusiva?}

El adjetivo inclusivo añadido al sustantivo educación (Jarque, 2016), lo que nos viene a decir es que tenemos que trabajar para conseguir que la educación escolar que ahora tenemos - heredera de unas formas de pensar y valorar la diversidad del alumnado en términos de categorías excluyentes y jerarquizadas; niños y niñas, alumnos buenos y malos, capaces y (dis)capacitados, payos y gitanos, autóctonos y migrantes, "normales y raros", ... (Ballard, 2012; Echeita, Simón, López y Urbina, 2014)-, NO es capaz de responder con equidad en relación a tres grandes tareas:

- Primero, acoger a TODO el alumnado, independiente de sus necesidades educativas -porque, si nadie dice lo contrario, todos tenemos igual dignidad y derecho a estar y compartir los espacios comunes donde se construye la ciudadanía-.

- Segundo, hacer que TODOS se sientan reconocidos, partícipes activos y personas queridas y estimadas por sus iguales y su profesorado. Ocurre que cuando la escuela acoge hoy a muchos de los que han estado fuera por mucho tiempo (por ejemplo, buena parte de la infancia con (dis)capacidad), no les ofrece a todas y a todos, oportunidades equiparables para que, en efecto, sean queridos y estimados por como son (no por su cercanía o lejanía a un determinado patrón de normalidad); para que construyan una identidad en positivo y no "deficitaria" o de menor valor, y para que se sientan parte de un grupo y tengan amistades y relaciones sociales significativas, alejando con ello el riesgo de marginación o peor aún, de maltrato por sus iguales (Calderón, 2014; Fernández Enguita, Gaete y Terrén, 2008).

- Y en tercer lugar, hablamos de educación inclusiva porque la educación que vemos desarrollarse cotidianamente en los centros educativos (desde infantil hasta la universidad) NO tiene suficientes estrategias, formas de organización y modos de enseñar y evaluar variados y diversifi- 
cados (Echeita, Simón y Sandoval, 2014), que permitan el aprendizaje, al más alto nivel y rendimiento posible, de TODO el alumnado y de forma personalizada (Coll, 2016), alejando con ello esa lacra de los altos índices de "fracaso escolar y de la escuela" que hoy alcanza, en algunos contextos, a más de una cuarta parte de la población escolar (Escudero y Martínez, 2012)

El enorme desafío que la educación inclusiva supone, entonces, es el de articular con equidad para TODO el alumnado las tres dimensiones referidas: acceder o estar presente en los espacios comunes/ordinarios donde todos se deben educar; participar, convivir y tener un bienestar acorde con la dignidad de todo ser humano y, finalmente, aprender y progresar en la adquisición de las competencias básicas necesarias para alcanzar una vida adulta de calidad, sin dejar a nadie atrás por razones, personales o sociales, individuales o grupales.

En definitiva, necesitamos poner el énfasis en el adjetivo "inclusiva" porque, sin menospreciar los progresos que se han producido, todavía tenemos una educación escolar muy excluyente en forma de segregación, de marginación y/o de fracaso escolar de muchas alumnas y alumnas a lo largo de su proceso educativo.

Bien podría decirse que este adjetivo es uno más - de momento digamos "el penúltimo" -, de los que hemos ido añadiendo a "la educación" a secas, a medida que nuestras ambiciones sociales se han hecho más fuertes y que han ido aflorando nuevos desafíos sociales. Otro de los adjetivos que necesita nuestra educación es por ejemplo, que contribuya a la sostenibilidad medioambiental de nuestro planeta (Echeita y Navarro, 2014; Ecologistas en Acción/MRPs, 2015)

\section{3. ¿De quién hablamos cuando hablamos de educación inclusiva?}

Casi desde el inicio ya he anticipado la respuesta a esta pregunta: hablamos de TODO el alumnado, sin exclusiones, ni restricciones, ni eufemismos. Esto es, no hablamos de TODOS para referirnos a la mayoría o a casi todos, sino que es un TODO $\mathrm{S}$ absoluto: pero ¿se refiere $\mathrm{Vd}$. también a ese alumnado que tiene amplias y extensas necesidades de apoyo para su desarrollo personal y social? ¿También, por ejemplo, a los alumnos y alumnas con discapacidades intelectuales o del desarrollo? Pues SI, también a ellos y a ellas. ¿O es que acaso son personas a las que no debamos considerar con igual dignidad y derechos que el resto? (Urien,2017). ¿Está escrito en la Declaración Universal de los Derechos Humanos que no lo sean? Pues NO y eso es lo que, como señalaba al inicio, ha ratificado la Convención de Derechos de las Personas con Discapacidad (UN, 2006).

Tener muy presente que hablamos de TODO el alumnado es necesario para prevenirnos, en primer lugar, del riesgo de seguir con políticas educativas, como buena parte de las actuales, cuyas actuaciones en materia de equidad se basan en una categorización del alumnado vulnerable, focalizando solamente en estos y en los centros escolares donde mayoritariamente se escolarizan las actuaciones y recursos compensatorios. Por cierto, esta es una forma de pensar muy habitual también en otras políticas que buscan reducir la exclusión (por ejemplo, en el ámbito laboral (Castell, 2004).

Pero es evidente que estamos ante un más que difícil proceso de transformación educativa (y social) que llevará tiempo y que supondrá un gran esfuerzo. No debe extrañarnos, entonces, que aquellos que más han vivido en sus carnes y con mayor crudeza procesos de segregación, marginación y mal/poco aprendizaje, sean los primeros en reclamar para sí políticas y prácticas inclusivas. Tal es el caso, sin duda alguna, de las personas en situación de (dis)capacidad (Campoy, 2013), quienes han hecho de la bandera de la inclusión (educativa, social, laboral) su emblema y Leitmotiv ${ }^{3}$.

Estos análisis deben servirnos para resaltar la necesidad de no identificar el movimiento internacional y los desafíos hacia una educación más inclusiva como solamente propios de ese determinado colectivo o grupo de alumnos que, a partir de la legislación vigente en España, reconocemos como alumnado con necesidades educativas especiales.

Ello sería tan inadecuado, injusto e improductivo como dejarles nuevamente en segundo plano a cuenta de que hablamos de TODO el alumnado, o porque hay otros alumnos o alumnas igualmente vulnerables que, sin embargo, son más numerosos (por ejemplo, el alumnado que vive en contextos sociales empobrecidos), o porque su realidad es más preocupante habida cuenta de las repercusiones sociales que se asocian a su fracaso/abandono escolar (delincuencia, abuso de drogas, marginalidad...)

\section{4. ¿Cuáles son los principales desafíos para el desarrollo del derecho a una educación inclusiva?}

Adoptar la perspectiva de los derechos en el ámbito de la educación inclusiva supone, necesariamente, trabajar para conseguir las condiciones que hagan posible su disfrute. De lo contrario, lo que se hace de facto es negar el ejercicio efectivo de los mismos. Esas condiciones escolares no son ni pueden ser las que han venido existiendo en la "gramática escolar" existente en nuestro sistema educativo (Echeita, Simón, Sandoval, 2016; Simón, et al, 2016).

Por ello, el principal desafío que, a mi juicio, debe acometerse en el marco de la nueva ley es el de crear las condiciones para que los centros educativos sean capaces de iniciar y sostener sistémicos procesos de mejora e innovación educativa para trasladar la educación inclusiva del olimpo de los deseos a la realidad de las aulas.

Para los centros educativos esta empresa colectiva, tiene a sus espaldas dos tareas irrenunciables:

- En primer lugar, reconocer las múltiples barreras (conjunto de factores que limitan o pueden llegar a limitar la presencia, el aprendizaje y la participación del alumnado) que existen actualmente en las culturas, las políticas y las prácticas educativas (derivadas de un las viejas forma de pensar y actuar, Echeita, Simón, López y Urbina, 2014). Los centros deberían estar llamados a revisar profundamente sus proyectos educativos y programas institucionales bajo el prisma de este análisis.

- La segunda tarea es transformar tales barreras en facilitadores - en esos mismos planos-, de una acción educativa con capacidad para personalizar la enseñanza ajustándose a la diversidad del alumnado y responder con equidad a sus necesidades educativas y aspiraciones. Ello requiere conocimientos no solo sobre el qué hacer (Coll, 2016), propiamente dicho, sino también sobre el cómo implementar los cambios necesarios, esto es, sobre cómo iniciar y hacer sostenibles (a largo plazo) los cambios educativos requeridos, un conjunto de saberes que se articulan alrededor

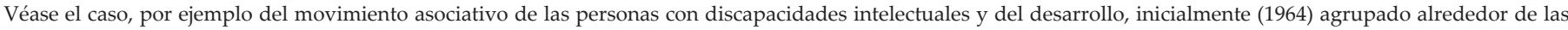
siglas FEAPS (Federación Nacional de Asociaciones Pro Subnormales) y actualmente (desde 2016), bajo el lema Plena Inclusión: http://www.plenainclusion.org/ 
de la investigación sobre eficacia de la mejora escolar (Murillo, Krichesky, 2012; Murillo, Krichesky, 2015).

Lo importante es que hoy tenemos los conocimientos, la experiencia y la capacidad para llevar a cabo estas dos tareas y, de hecho, la literatura especializada está repleta de conocimientos rigurosos para guiarnos en este proceso. Valgan de muestra dos botones; Booth, y Ainscow, (2015) y UNESCO/BIE (2016). Son conocimientos que confluyen en una idea clave: es posible hacerlo. Hay administraciones educativas, centros, y profesorado que han sido capaces de ponerse en marcha y empezar a recorrer el camino que media entre sus ambiciones y su realidad. No son perfectos, no lo son eternamente ni en todas las circunstancias, pero tampoco son anecdóticos.

Pero también sabemos que saber no es suficiente. En efecto, lo cierto es que tenemos conocimientos, pero lo que no está claro que tengamos es la voluntad política suficiente (en los distintos niveles; nacional, autonómico y local en los que esta se distribuye), para movilizar el saber disponible y para enfrentarse a las múltiples turbulencias y resistencias que este proceso va a originar en los sistemas educativas establecidos.

El carácter sistémico de este proceso nos alerta de la necesidad de generar muchas condiciones y políticas que tienen que alinearse de modo coherente para que se pueda avanzar hacia la meta señalada; condiciones y políticas en materia de financiación, de infraestructura (sin ir más lejos, en relación con la accesibilidad de espacios físicos y virtuales); en materia de revisión del currículo (menos sobrecargado de contenidos y más cuidadoso con el hecho de que los que se consideren imprescindibles contribuyan al desarrollo de todas las inteligencias/competencias que se deben enseñar y no solo algunas); de ordenación de las enseñanzas (en tareas tan sensibles como los procesos de transición entre etapas o los criterios de promoción y de titulación - si es que esta fuera imprescindible, cosa que no todo el mundo defiende ${ }^{4}$ ), etc.

No estoy en condiciones de analizarlas todas, pero sí de enfatizar algunas imprescindibles:

$\checkmark$ Asentar una visión compartida, fuertemente arraigada en un conjunto claro de valores y principios educativos inclusivos (Booth, 2006; Booth y Ainscow, 2015; Escudero, 2006; Extebarría, 2005):

o La dignidad intrínseca de todo ser humano por encima de las diferencias que configuran nuestra diversidad.

o La justicia que vela contra la discriminación y el trato desigual.

o La acción benefactora: "procurar el bien de quien me siento responsable y el cuidado, en particular, de aquellos más vulnerables"

o La responsabilidad, para acometer los cambios o mejoras que reduzcan las injusticias y promuevan valores deseados.

$\checkmark$ Extender una fuerte capacidad entre todos los miembros de la comunidad educativa para reflexionar sobre los "sistemas de prácticas" en los que se deben enraizar los valores compartidos y que, por ello, sostienen las "culturas morales" que bien cabría llamar inclusivas (Puig Rovira et al, 2012).

$\checkmark$ Desarrollar un liderazgo escolar sólido en el que confluyan tres dimensiones básicas:

o un liderazgo pedagógico, un liderazgo distribuido y un $l i$ derazgo para la justicia social (Bolívar, López y Murillo, 2013). Un liderazgo que, en definitiva, permita crear y sostener las condiciones internas que sostienen las culturas inclusivas (Murillo, Krichesky, Castro, y Hernández, 2010).

$\checkmark$ Construir fuertes y consistentes culturas, políticas y prácticas colaborativas a varios niveles; dentro del centro escolar y entre centros escolares; entre el profesorado y entre el alumnado, y entre unos y otros con las familias y el contexto local (Ainscow y West, 2008).

$\checkmark$ Formar desde el inicio a todo el profesorado con una firme convicción de que "la capacidad de aprender de todos sus estudiantes puede cambiar y ser cambiada a mejor como resultado de lo que él/ella puede hacer en el presente" (Hart et al., 2004).

o Es la concepción "transformadora" de la enseñanza y el aprendizaje, opuesta a la visión determinista que se traduce en una concepción sobre la capacidad de aprendizaje condicionada genéticamente y que se refleja en el C.I. de cada estudiante.

$\checkmark$ Garantizar que todo el profesorado (tanto el que esté llamado a enseñar en educación infantil, como en educación primaria y en educación secundaria), egrese de su formación inicial, con las competencias necesarias para sentirse y desempeñarse como profesor o profesora de TODO el alumnado, algo que no parece que estemos consiguiendo a pesar de las reformas recientes en sus planes de formación (Echeita, 2012; Izusquiza, Echeita, y Simón, 2015).

$\checkmark$ Una formación inicial y permanente que debe servir para afianzar las competencias emocionales propias de un profesorado empático que sabe y quiere escuchar la voz de su alumnado, que confía en su capacidad para implicarse en su proceso de aprendizaje y en su capacidad para decirnos sin ambages lo que le hace sentirse mal y no aprender (Vaello, 2009; Susinos y Ceballos, 2012).

$\checkmark$ Aprender a implementar un desarrollo institucional bien pensado y planificado que, por ello, pueda ser sostenible en el tiempo y con capacidad para aguantar las innumerables turbulencias de un proceso constantemente amenazado $\mathrm{y}$, en todo caso, sujeto a las cambiantes circunstancias de su propio "ecosistema": la crisis socioeconómica, la falta de recursos, y de apoyos, la desmoralización._Ainscow, Dyson, West y Goldrick, 2013).

Estas condiciones en particular no son un "milagro" que unos centros reciben y otros no, o "cualidades personales" que algunas profesoras o profesores tienen por azar. Todas ellas son capacidades que se pueden aprender y al alcance de la mayoría del profesorado a través de procesos de formación continua, asesoramiento y apoyo institucional. No me cabe duda de que si la nueva ley no contempla de forma prioritaria estas acciones de formación permanente, asesoramiento psicopedagógico y apoyo a los centros en sus procesos de mejora e innovación estaremos, de nuevo, ante la frustración de ver incumplidas algunas buenas intenciones. Una frustración que, por otra parte, es bastante común en los cinco continentes (Artiles, Kokleski y Waitoller, 2015).

Ciertamente implementar este tipo de políticas de apoyo para la mejora es costoso y bastante difícil cuando, al mismo tiempo, es muy fácil convertir a las víctimas de su ausencia - esto es a los "malos alumnos" de los que nos hablaba Marchesi (2004)-, en culpables de su mal comportamiento, marginación o fracaso escolar.

\footnotetext{
Ver la opinión de Julio Carabaña al respecto: http://eldiariodelaeducacion.com/blog/2017/05/16/julio-carabana-defiende-la-eliminacion-del-titulo-de-eso/
} 


\section{Colaboración y apoyo para generar esperanza.}

Creo que en todo el recorrido de este texto no he dejado de señalar en los momentos oportunos que este gran desafío de avanzar hacia una educación más inclusiva, es un proceso complejo, éticamente controvertido, difícil y cuajado de dilemas. Soy idealista respecto a la meta que perseguimos, pero vivo con los pies en la tierra y conozco bien las turbulencias y dificultades que este proceso acarrea.

Por ello, comparto plenamente con otros autores que el principal ingrediente en estas circunstancias, para no sucumbir al desaliento que estas dificultades acarrean, es emocional. No es otro que construir colectivamente un fuerte sentido de esperan$z a$. Pero entendida esta no como un sentimiento melifluo de que "las cosas, tarde o temprano irán bien", sino como la capacidad de "no entrar en pánico ante tales dificultades" (Fullan, 2001). Y esa emoción se construye sobre la base de una fuerte cultura colaborativa al interior de los centros escolares, entre los centros escolares y de estos con su comunidad local. Esta es una de las condiciones que anteriormente he señalado y sobre la que creo necesario decir algo más.

Esa cultura colaborativa se construye con políticas y prácticas de muy distinto tipo. Ya es un tópico (pero no tanto una realidad generalizada), hablar de la importancia del trabajo cooperativo entre el alumnado. Una colaboración y apoyo entre el alumnado que se debe construir con conocimiento y paciencia, que admite muchos formatos (grupos cooperativos, tutoría entre iguales, grupos interactivos, alumnos mediadores, ...) y que aguanta poco las improvisaciones o los calentones de las acciones puntuales que a la larga no se sostienen en el tiempo (Pujolas, Lago y Naranjo, 2013; Topping, Buchs, Duran y Van Keer 2017).

Menos comunes, pero no por ello menos importantes, son las estrategias de colaboración y apoyo mutuo entre el profesorado cuando, sin embargo, sabemos bien del papel que, por ejemplo, las estrategias conocidas como "estudio de lecciones" ("lessons study") pueden desempeñar a la hora de diseñar e implementar prácticas educativas inclusivas (Messiou, t al, 2016).

Finalmente, y sin pretender con ello ser exhaustivo a este respecto, es obvio que las familias son una hebra y un nudo central a la hora de tejer esta cultura colaborativa. Se necesita mucha inteligencia emocional y modelos claros de relación con las familias para que estas se conviertan en el principal aliado de los equipos docentes a la hora de iniciar y sostener proyectos educativos inclusivos (Simón, Giné y Echeita, 2016).

Reforzar esta cultura colaborativa al interior de los centros y el trabajo en red entre centros escolares y entre estos y su comunidad educativa y local (Parrilla, Nuñez y Sierra, 2013), está llamada a ser una estrategia crítica en este proceso. Lo que tengo claro también es que esta cultura colaborativa encaja mal con las crecientes tendencias a estimular la competitividad entre centros (como entre Comunidades Autónomas o países) a través del desajustado papel que están teniendo las evaluaciones de rendimiento nacionales e internacionales. Creo que es posible aunar rendición de cuentas, con políticas de colaboración y trabajo en red que faciliten la mejora de la equidad. El trabajo desarrollado por el profesor Mel Ainscow, a través del proyecto "Great Manchester" es una política inspiradora de la que mucho se puede aprender (Ainscow, 2016).

\section{Dilemas y quebrantos en la educación inclusiva.}

De lo dicho hasta aquí bien cabría deducir una valoración, hasta cierto punto positiva, del desarrollo de la educación inclu- siva en nuestro país. Sobre todo, hemos visto que la educación inclusiva ha pasado de ser un principio a un derecho y este cambio de estatus, a la larga, será determinante a mejor.

No cabe duda que, por otra parte, muchos centros por toda la geografía del país han ido adoptando, políticas y prácticas más inclusivas, mostrando con su quehacer cotidiano que, como decía anteriormente, es posible hacerlo.

Y no cabe duda de que, por ejemplo, en términos de presencia en los centros ordinarios del alumnado más vulnerable, se han producido avances más que significativos: casi ningún niño o niña está hoy sin escolarizar y tiene oportunidades de aprendizaje desde los 3 hasta los 16/18 años; ya no hay escuelas segregadas para niñas, ni otras para niños o niñas gitanos o para aquellos que vienen de otros países; y entre aquel alumnado considerado con necesidades educativas especiales, cuyo destino habitual era el de estar escolarizados en centros segregados (Aulas o Colegios Educación Especial, CEE), hoy las tasas de integración escolar se sitúan, como media, alrededor de un $70 / 80 \%$, si bien es cierto con una significativa variabilidad entre Comunidades Autónomas (MECD, 2015). Si pensamos que, en Alemania, por ejemplo, o en Holanda este mismo alumnado sigue estando mayoritariamente escolarizado en Colegios Especiales, sería injusto no reconocer estos hechos como positivos. Es cierto que las situaciones de maltrato entre iguales siguen ahí resistiéndose a menguar, pero también que "los valores de sentido de pertenencia al centro educativo en los alumnos españoles son los más altos de todos los países participantes en PISA" ${ }^{\prime 5}$

Alguien podría decir, entonces, que tenemos razones para sonreír. Pero creo que también tenemos muchas razones para la preocupación y, sobre todo, algunas familias en particular - garantes del derecho de sus hijos e hijas-, no tienen nada que celebrar y mucho que reclamar y lamentar (Doménech, 2017).

Alguien dijo del amor que "si no crece, decrece". Tal vez quepa decir lo mismo del compromiso hacia una educación más inclusiva. $\mathrm{Y}$ en estos momentos, mi impresión es que decrece. Decrece porque no crecen (más bien están ausentes o son muy débiles), las políticas de las administraciones dirigidas a promover, financiar, acompañar y sostener los amplios y sistémicos procesos de mejora escolar e innovación educativa de los que precisa, sí o sí, el desarrollo de una educación inclusiva.

Sin estos, solo cabe esperar que lo centros que se incorporen a este compromiso lo hagan con proyectos educativos inclusivos incompletos, débiles y a la larga, fallidos. Centros que, como ya ocurre en la actualidad, se comprometen con "la inclusión" de algunos, pero no con la de otros alumnos o alumnas por ser "especiales" o porque sus necesidades educativas requieren de complejos apoyos; centros que "incluyen" en educación infantil y primaria, pero que luego "invitan", sic, a buena parte de ese mismo alumnado a marchar cuando llega la temida secundaria (Doménech, 2017). O centros de secundaria donde esos alumnos y alumnas más vulnerables, están viviendo auténticas situaciones de marginación y, en el mejor de los casos, aprendiendo poco.

Y como la capacidad de respuesta inclusiva de los centros no mejora, lo que aumenta es la proliferación de normas, medidas, dispositivos y centros especiales, más o menos segregadores y excluyentes; para "atender a la diversidad", como eufemísticamente se denominan. Y si llegado el caso alguno de estos "dispositivos" funciona bien y ofrecen una respuesta educativa digna y aceptable, o en su caso una "segunda oportunidad" (AA.VV, 2017) para entrar al mundo adulto y laboral con menos riesgo, entonces hacen, a la larga, un flaco favor a la mejora de la equidad en

https://www.oecd.org/pisa/PISA2015-Students-Well-being-Country-note-Spain-Spanish.pdf 
los centros ordinarios, pues estos dejan de sentir la presión para el cambio y, además, pueden justificar que no es necesario ese esfuerzo porque objetivamente es en esos dispositivos/grupos/ aulas/centros "especiales" o de "segunda oportunidad" (E2O) donde ese alumnado aprende y emocionalmente está mejor, lo cual es, en muchos casos, cierto, al menos durante ese tiempo de escolarización.

En el marco de este panorama, muchas familias de estos alumnos sufren y se enfrentan a un dilema moral que les abrasa emocionalmente, en particular en los casos del alumnado que consideramos con necesidades educativas especiales. De muestra vale un botón:

Seremos invisibles. (Publicado el15 de febrero de 2017 En El Margen)

Ares abandonará la escuela ordinaria a final de curso, si conseguimos plaza en el Centro de Educación Especial que queremos.

Creemos en la inclusión, pero la inclusión no cree en nosotros. Tenemos leyes garantistas con presupuestos excluyentes que convierten automáticamente a las leyes en excluyentes. Tenemos buena voluntad, ganas, ilusión, pero muchas veces falta todo lo demás. Y todo lo demás, muchas veces, son demasiadas cosas.

Creemos en la inclusión, pero no a toda costa. Creemos en la inclusión si todos (sociedad, escuela, leyes, presupuestos, personas...) remamos en la misma dirección: la de incluir al que ya está, aunque sea diferente.

Nos marchamos de la educación ordinaria convencidos, aunque tristes. Tristes porque el sistema, para los niños con las dificultades que tiene Ares, te incluye con una mano, mientras te muestra con la otra la puerta de salida.

Ares desparecerá de su entorno. El que le pertenece. Dejará de aprender algunas cosas y de enseñar muchas más a los que la rodean. El sistema conseguirá que todo sea más uniforme. Y que seamos invisibles. (En El Margen. Blog:

https://enelmargenn.wordpress.com/2017/02/15/seremos-invisibles/)

Como bien refleja la familia de Ares, ellos como otros se habían alegrado con la noticia del reconocimiento de que sus hijos o hijas tenían derecho a una educación inclusiva de calidad. Es probable que hayan asistido a alguna conferencia, congreso o seminario sobre el tema, donde una persona, tal vez como yo, les haya explicado y desmenuzado lo que significa ese derecho, haciéndoles sentir que el futuro de su hijo/a podía ser esperanzador. Pero lo cierto es que no lo es para muchos (aunque si lo ha sido para otros).

$\mathrm{Y}$ ahora muchos de ellos no saben qué hacer y algunos de nosotros no sabemos que decirles; ¿Mantenerse en centros ordinarios donde no se está ofreciendo una respuesta educativa de calidad acorde a las necesidades específicas de sus hijos/as? ¿Retirarse a un centro de educación especial donde puede ser que, al menos, estén más tranquilos y mejor "atendidos" provisionalmente? ¿Pelear por sus derechos o resignarse a la situación, sabiendo, en todo caso, que "las peleas legales son costosas en tiempo y dinero" y que el tiempo pasa sin detenerse a esperar la resolución de estos dilemas? ¿Pensar en su "hoy de niño" o en su "futuro como ciudadano adulto", en una sociedad que quisiera que fuera inclusiva?

Entiendo que algunas de esas familias nos reprochen a los académicos que no nos implicamos profundamente con el presente de sus hijos, mientras que, sim embargo, hacemos de sus preocupaciones y anhelos el contenido de nuestras propuestas a futuro a través de nuestras investigaciones y publicaciones.
Pero también sabemos que los procesos de cambio llevan mucho tiempo y que mientras estos llegan con cierta solidez (¡si llegan!), las malas condiciones para la escolarización de algunos alumnos o alumnas (cuyas características personales son particularmente desafiantes en relación a la "gramática escolar existente"), generarían un quebranto continuo para ellos, su profesorado y sus compañeros, cuyos derechos tampoco pueden ser olvidados. Uno entiende que, incluso aquellos responsables de las políticas educativas comprometidos con esta meta, estén temerosos ante propuestas más inclusivas.

Algo similar les ocurre a algunos orientadores y orientadoras que trabajan particularmente en las etapas de atención temprana, infantil y primaria; se saben partícipes de un proceso de discriminación (el que les obliga por mandato de las normas establecidas a elaborar informes y dictámenes de escolarización mediante los cuales de derivan a algunos alumnos a CEE), algo que es contrario a lo establecido en la Convención de los Derechos de las Personas con Discapacidad y también a sus convicciones y su código deontológico profesional. $\mathrm{Ob}$ viamente otros viven sin preocupaciones, instalados en sus viejos modelos y prácticas de orientación y esta tensión, ni les inmuta.

No tengo muy claro qué hacer y también me siento aturdido ante estos dilemas. Pero si tengo claro que todos, no ya como educadores sino como ciudadanos, deberíamos estar informados de la contradicción que supone haber establecido con todo el rigor de la ley un derecho de gran transcendencia para la sociedad (el derecho a una educación inclusiva) y luego saber que no solo no se cumple, sino que podríamos estar en claro riesgo de ir hacia atrás y de convertirlo en beneficencia o "un desecho":

“2. m. Cosa que, por usada o por cualquier otra razón, no sirve a la persona para quien se hizo." (Diccionario de la RAE).

¿Habremos ido demasiado lejos en el intento de acercar el sueño de una educación inclusiva a nuestras aulas, en particular, en las de los centros de educación secundaria? ¿Debemos recalibrar esta meta? Esto es, la educación inclusiva ¿Debe ser solo una aspiración reducida para algunos de los muchos alumnos o alumnas vulnerables, durante algún tiempo, en algunos centros que voluntariamente estén dispuestos a ello y, obviamente, en algunos países ricos?

¿Le diremos a las familias que están manteniendo una lucha vital por el derecho de sus hijos e hijas a una educación inclusiva que su lucha es "utópica" y que se resignen a la situación de opresión y desventaja que les ha tocado vivir?

Educación inclusiva; sonrisas y lágrimas.

\section{Referencias}

AA.VV. (2017) Escuelas de segunda oportunidad. Tema del mes. Cuadernos de Pedagogía, 478, 46-79

Ainscow, M. (2016). Struggles for Equity in Education. Londres. Routledge

Ainscow, M, Dyson, A., West, M. y Goldrick, S. (2013). Promoviendo la equidad en la escuela. Revista de Investigación Educativa. Número monográfico, 11(3), 44-56.

Ainscow, M. y West, M. (2008). Mejorar las escuelas urbanas. Liderazgo y colaboración. Madrid: Narcea

BIE/UNESCO (2016). Reaching Out to All Learners: a Resource Pack for Supporting Inclusive Education. Ginebra: BIE/UNESCO.

Bolívar, A, Lopez, Y, y Murillo, J. (2013). Liderazgo en las instituciones educativas. una revisión de líneas de investigación. Revista Fuentes, 14, 15-60. 
Booth, T. (2006). Manteniendo el futuro con vida; convirtiendo los valores de la inclusión en acciones. En M.A. Verdugo y F.B. Jordán de Urríes (Coords.), Rompiendo inercias. Claves para avanzar. (pp. 211-217). Salamanca: Amarú.

Booth, T. y Ainscow. M. (2015). Guía para la Educación Inclusiva. Desarrollando el aprendizaje y la participación en los centros escolares. Madrid: OEI/FUHEM

Calderón, I. (2014). Educación y esperanza en las fronteras de la discapacidad. Madrid: CERMI

Campoy, I (2007). La educación de los niños en el discurso de los derechos humanos En, I. Campoy (Ed). Los derechos de los niños: perspectivas, jurídicas y filosóficas, (pp.149-201). Madrid: Dykinson

Campoy, I. (2013). Estudio sobre la situación de los niños y las niñas con discapacidad en España. Madrid: UNICEF.

Castel, R. (2004). Encuadre de la exclusión En S. Karsz (2004). La exclusión: bordeando sus fronteras. Definiciones y matices (pp. 5758), Barcelona: Gedisa.

Col, C. (2013). El currículum escolar en la nueva ecología del aprendizaje. Aula de Innovación, 219, 31-36.

Coll, C. (2016). La personalización del aprendizaje escolar El qué, el por qué y el cómo de un reto insoslayable. En J.M. Vilalta (DIr). Reptes de l'educació a Catalunya. Anuari d'Educació 2015. (pp. 36- ) Barcelona: Fundació Jaume Bofill. Traducción de Iris Merino.

Doménech, A. (2017). Sorteando barreras hacia la inclusión: Una historia de vida. Castellón: Tesis doctoral no publicada. Facultad de Ciencias Humanas y Sociales. Universidad Jaume I.

Echeita, G. (2012). Competencias esenciales en la formación inicial de un profesorado inclusivo. Un proyecto de la Agencia Europea para el Desarrollo de las Necesidades Educativas Especiales y la Educción Inclusiva. Tendencias Pedagógicas, 19, 7-24.

Echeita, G. (2013). Inclusión y exclusión educativa. De nuevo, "voz y quebranto". REICE. Revista Iberoamericana sobre Calidad, Eficacia y Cambio en Educación, 11(2), 99-118.

Echeita, G.; Simón, C.; López, M., y Urbina, C. (2013). Educación inclusiva. Sistemas de referencia, coordenadas y vórtices de un proceso dilemático. En M.A. Verdugo y R. Shalock (Coordinadores). Discapacidad e inclusión. Manual para la docencia. (pp. 307-328). Salamanca: Amaru

Echeita, G. y Navarro. D. (2014). Educación inclusiva y desarrollo sostenible. Una llamada urgente a pensarlas juntas. EDETANIA 46, 141-161,

Echeita, G. Simón, C. y Sandoval, M. (2016). Notas para una pedagogía inclusiva en las aulas. En, Actas de las IV Jornadas Iberoamericanas de Síndrome de Down. Salamanca: INICO.

Ecologistas en Acción y MRPs (2015). 99 preguntas y 99 experiencias para aprender a vivir en mundo justo y sostenible. Madrid: MRPs y Ecologistas en Acción.

Etxeberria, X. (2005). Aproximación ética a la discapacidad. Bilbao. Ediciones de la Universidad de Deusto

Escudero, R. (2006). Compartir propósitos y responsabilidades para una mejora democrática de la educación. Revista de Educación, 339, 19-42

Escudero, R. y Martínez, B. (2012). Las políticas de lucha contra el fracaso escolar: ¿programas especiales o cambios profundos del sistema y la educación? Revista de Educación, número extraordinario, 174-193

Fernández Enguita, M., Gaete, J.M. y Terrén, E. (2008). ¿Fronteras en las aulas? Contacto transcultural y endogamia en las interacciones del alumnado. Revista de Educación, 345, 157-181

Fullan, M. (2001). Emoción y esperanza: conceptos constructivos para tiempos complejos En A. Hargreaves (Coord.). Replan- tear el cambio educativo. Un enfoque renovador (pp. 296-317). Madrid: Amorrortu.

Hart, S., Dixon, Ab, Drummond, M.J., y McIntyre, D. (2010). Learning without limits. Maidenhead: Open University Press

Izusquiza, L., Echeita, G. y Simón, C. (2015). La percepción de estudiantes egresados de magisterio en la universidad autónoma de Madrid sobre su competencia profesional para ser "profesorado inclusivo": un estudio preliminar. Tendencias Pedagógicas, 26, 197-216

Jarque, J.M. (2016). ¿De qué hablamos de inclusión o de escuela inclusiva? Un esbozo de la cuestión en Cataluña. Dosier Graó, $1(1), 42-46$

Lema, C. (2009). El impacto en la educación de la Convención de la ONU sobre los derechos de las personas con discapacidad. En, M.A. Casanova y M.A. Cabra de Luna (Coords). Educación y personas con discapacidad. Presente y futuro. (pp. 31-65). Madrid: Funación ONCE

Marchesi, A. (2004). Que será de nosotros, los malos alumnos. Madrid: Alianza Editorial.

Ministerio de Educación, Cultura y Deporte (2015). Estadística de las enseñanzas no universitarias. Alumnado con necesidad específica de apoyo educativo curso 2014-2015. Nota Resumen.

Messiou, K., Ainscow, M., Echeita, G., Goldrick, S., Hope, M., Paes, I., et al. (2016). Learning from differences: a strategy for teacher development in respect to student diversity. School Effectiveness and School Improvement, 27(1), 45-61

Murillo, F.J.; Krichesky, G., Castro, A.M. y Hernández, R. (2010). Liderazgo para la inclusion escolar y la justicia social. Aportaciones de la investigación. Revista Lationamericana de Educación Inclusiva, 4(1), 169-186.

Murillo, F.J. y Krichesky, G. (2012). El Proceso del Cambio Escolar. Una Guía para Impulsar y Sostener la Mejora de las Escuelas. REICE. Revista Iberoamericana sobre Calidad, Eficacia y Cambio en Educación, 10(1), 26-43.

Murillo, F.J. y Krichesky, G. (2015). Mejora de la Escuela: Medio siglo de lecciones aprendidas. REICE. Revista Iberoamericana sobre Calidad, Eficacia y Cambio en Educación, 13(1), 69-102.

Parrilla, A., Muñoz-Cadavid, M.A. y Sierra S. (2013). Proyectos educativos con vocación comunitaria. Revista de Investigación Educativa. Número monográfico, 11(3), 15-31

Puig Rovira, J.M., Doménech, I., Gijón, M., Martín, X., Rubio, L. y Trilla, J. (2012). Cultura moral y educación. Barcelona: Graó.

Sandoval, M. (2011). Aprendiendo de las voces de los alumnos y alumnas para construir una escuela inclusiva. REICE, Revista iberoamericana sobre calidad, eficacia y cambio en educación. 9 (4).

Simón, C., Giné, C. y Echeita, G. (2016). Escuela, familia y comunidad: Construyendo alianzas para promover la inclusión. Revista Latinoamericana de Inclusión Educativa, 10(1), 25-42.

Simón, C., Sandoval, M, Echeita, G, Calero, C, Nuñez, B, de Sotto, P, Pérez, M. y García, A.B. (2016). Transformando la "gramática escolar" para ser más inclusivos. La experiencia de tres centros educativos. Contextos, Revista de Educación, 19, 7-24

Slee, R. (2012). La escuela extraordinaria. Exclusión, escolarización y educación inclusiva. Madrid: Morata

Susinos, T y Ceballos, N. (2012). Voz del alumnado y presencia participativa en la vida escolar. Apuntes para una cartografía de la voz del alumnado en la mejora educativa. Revista de Educación, 359, 24-44

Topping, K., Buchs, C., Duran, D., \& Van Keer, H. (2017). Effective peer learning: From principles to practical implementation. Londres: Routledge.

Touraine, A. (2005). ¿Podremos vivir juntos? Iguales y diferentes. Madrid: PPC 
UN (2006). Convención de las Naciones Unidas sobre los derechos de las personas con discapacidad.

UN. Comité de Derechos de las Personas con Discapacidad (2016). Observación General 4. Derecho a una educación inclusiva.

UN (2016). Objetivos de desarrollo sostenible, 2030.

UNESCO/BIE (2008). Informe de la 48 Conferencia Internacional. La educación inclusiva, el camino hacia el futuro.
UNESCO (y otras organizaciones) (2016). Declaración de Incheon. Urien, T. (2017). Del reconocimiento legal al reconocimiento efectivo. De la igual dignidad como un derecho de la persona con discapacidad intelectual o del desarrollo. Un proceso que nos interpela. Siglo Cero, 47 (2), 43-62.

Vaello, J. (2009). El profesor emocionalmente competente. Un puente sobre aguas turbulentas. Barcelona: Graó. 